\title{
Adaptive fluid-infused porous films with tunable transparency and wettability
}

\section{Citation}

Yao, Xi, Yuhang Hu, Alison Grinthal, Tak-Sing Wong, L. Mahadevan, and Joanna Aizenberg. 2013. "Adaptive Fluid-Infused Porous Films with Tunable Transparency and Wettability." Nat Mater 12 (6) (April 7): 529-534. doi:10.1038/nmat3598.

\section{Published Version}

doi:10.1038/nmat3598

\section{Permanent link}

http://nrs.harvard.edu/urn-3:HUL.InstRepos:27417438

\section{Terms of Use}

This article was downloaded from Harvard University's DASH repository, and is made available under the terms and conditions applicable to Other Posted Material, as set forth at http:// nrs.harvard.edu/urn-3:HUL.InstRepos:dash.current.terms-of-use\#LAA

\section{Share Your Story}

The Harvard community has made this article openly available.

Please share how this access benefits you. Submit a story.

Accessibility 


\title{
Adaptive fluid-infused porous films with tunable transparency and wettability
}

\author{
Xi Yao ${ }^{1,2}$, Yuhang Hu${ }^{1,2}$, Alison Grinthal1, Tak-Sing Wong1,2, L. Mahadevan',2,3, and Joanna \\ Aizenberg1,2,3 * \\ ${ }^{1}$ School of Engineering and Applied Sciences, Harvard University, Cambridge, Massachusetts 02138, USA \\ ${ }^{2}$ Wyss Institute for Biologically Inspired Engineering, Harvard University, Cambridge, Massachusetts 02138, USA. \\ ${ }^{3}$ Kavli Institute for Bionano Science and Technology, Harvard University, Cambridge, Massachusetts 02138, USA.
}

*To whom correspondence should be addressed. Email: jaiz@seas.harvard.edu

Materials that adapt dynamically to environmental changes are currently limited to two-state switching of single properties, and only a small number of strategies that may lead to materials with continuously adjustable characteristics have been reported $^{1-3}$. Here we introduce adaptive surfaces made of a liquid film supported by a nanoporous elastic substrate. As the substrate deforms, the liquid flows within the pores causing the smooth and defect-free surface to roughen through a continuous range of topographies. We show that a graded mechanical stimulus can be directly translated into finely tuned, dynamic adjustments of optical transparency and wettability. In particular, we demonstrate simultaneous control of the film's transparency and its ability to continuously manipulate various low-surface-tension droplets from free-sliding to pinned. This strategy should make possible the rational design of tunable, multifunctional adaptive materials for a broad range of applications. 
Current dynamic materials rely on a stimulus-triggered switch in their chemistry and/or morphology to produce a change in functional properties. Surface topography in particular has become a promising variable for controlling a wide range of unique reflective ${ }^{4}$, structural color ${ }^{5}$, wettability ${ }^{6,7}$, adhesive ${ }^{8,9}$, bacterial and cell patterning ${ }^{10}$, liquid transport11-13, and other functions, and is an increasingly appealing target for adaptive manipulation. Hybrid materials that incorporate distinct reconfiguring structural elements-and harness the combinatorial potential and interplay between complementary materials-are proving to be an especially versatile strategy for expanding the responsive repertoire $^{3}$. At the same time, the fine tuning of surface structure and chemistry that is necessary to access the delicate and complex states required for many properties-a difficult feat for fabrication by any means-is beyond the reach of the typically discontinuous switching mechanisms. Moreover, the inevitable and uncontrollable defects and heterogeneities of solid materials are incompatible with some of the most highly desired functions, such as optical precision and effective repulsion of low-surface-tension liquids $^{14}$.

Introducing a liquid into the surface offers a way out of these constraints. Dynamic fluid surfaces are a ubiquitous component of natural adaptive systems, from tear films on eyes ${ }^{15}$ to the mucous lining of lung and stomach ${ }^{16}$, to the light-adaptive skeletons of brittle stars ${ }^{17}$ and the responsive skin of cephalopods ${ }^{18}$. The fluid's ability to flow and configure itself around a variety of surfaces provides unique responsive mechanisms not possible with solid materials alone, yet synthetic adaptive materials design rarely goes beyond soft solids in the search for malleable surfaces. As we have recently shown, a liquid infused in a rigid porous substrate develops a continuous, defect-free, nearly atomically smooth overcoating film that shows superior surface properties in many realms, from omniphobicity to frost and biofouling prevention ${ }^{19-22}$. Here we demonstrate a completely new set of possibilities for adaptive behavior that arises from the variable interfacial properties associated with a liquid that impregnates a soft textured solid. While a fluid can flow, redistribute, and reconfigure in the textured solid, its configuration is eventually limited by the volume of available liquid and the substrate morphology, topography and space accessible for liquid infiltration ${ }^{23,24}$. Replacing the rigid substrate by an elastically responsive one and pairing 
this with the lubricant allows us to create a versatile, tunable, fully adaptive system capable of accessing a range of topographies and multifunctional surface properties via the interplay among elastic deformation, fluid flow, and liquid-solid interactions.

The adaptive behavior of the material developed in this work is based on one central concept: any deformation of the substrate that changes the pore size will cause the liquid to respond. As a result, fluid from the coating layer will be drawn into (out of) the expanding (contracting) pores, altering surface topography (Fig. 1a). This response can be quantitatively predicted by relating the induced pressure change to the substrate deformation and the interfacial tension. Initially the pressure of the infused liquid is balanced with the outside pressure. When a mechanically sensitive substrate is subject to a tensile stress $\sigma$, the pressure of the liquid inside the porous matrix decreases instantaneously, with the pressure drop $\Delta P=\sigma / 3 .{ }^{25-27}$ This causes the liquid interface to cave inwards until an equilibrium state is reached; then the pressure difference is balanced by capillary forces at the interface, with $\Delta P=2 \gamma / r_{\mathrm{m}}$, where $\gamma$ is the interfacial energy between the infused liquid and the outside media and $r_{\mathrm{m}}$ is the radius of the meniscus curvature (Fig. 1a). When the tensile stress is released, the pressure is released and the liquid moves outwards, forming a flat interface again. Thus the hybrid material is expected to provide a dynamic fluidic interface that can be reversibly varied between a smooth and textured one in response to stimuli sensed by the substrate.

We built an example of such a material by combining a low-surface-energy fluid that infiltrates a stretchable porous matrix on an elastic base (Fig. 1b). As the porous matrix, we chose Teflon membrane (composed of nanofiber networks, $\sim 45 \mu \mathrm{m}$ thick, average pore size $200 \mathrm{~nm})$ that can absorb and hold fluids. An elastic PDMS membrane ( $\sim 0.511 .5 \mathrm{~mm}$ thick) was attached to the porous Teflon membrane using a thin layer of PDMS oligomer as adhesive, such that stretching the base stretches the porous matrix while maintaining the mechanical integrity of the system (Supplementary Fig. S1-S2). A perfluorinated liquid (for example, DuPont Krytox 103 perfluoropolyether) that can wet and wick into the porous membrane was selected as the fluid lubricant layer. The surface energy match between the solid matrix and the lubricant, together with the matrix roughness and the lubricant's 
immiscibility with most other liquids, ensures that the lubricant will stably adhere to the matrix ${ }^{19}$. The resulting system integrates the characteristics of the elasticity, roughness and porosity of the substrate with a liquid lubricant's ability to flow and redistribute through and on it.

A finite element calculation indicates that applying a tensile stress to this system induces a pressure drop throughout the porous matrix consistent with our simple scaling theory above (see Supplementary for details). As shown in Figure 1c, a negative pressure field is built up in the membrane. In agreement with this prediction, our experimental observations indicate that this pressure drop drives the overcoating fluid to retreat into the pores. Macroscopically, as the surface changes from flat to rough, the optical properties are expected to change. Accordingly, a specular reflection is observed when the material is in the relaxed state and becomes diffuse when strain exceeds a critical value (Movie 1), indicating a transition from a smooth to a rough surface. This transition also causes a change in the material's ability to transmit light. As shown in Figure 1d, the liquid-infused matrix exhibits enhanced light transmission compared to the opaque porous Teflon membrane, due to the liquid's ability to fill the air pockets in the membrane and effectively reduce the light scattering at the surface/air interface. When the infused membrane is stretched, the transparent surface becomes opaque, as the liquid redistribution under membrane stretch generates partially air-filled pores (see insets in Fig. 1d).

The sensitivity of the film surface properties to matrix deformation makes it possible to finely tune the optical behavior over a continuous, wide range in response to stimuli. The commercial porous Teflon is constructed from nanofiber networks forming interconnected pores of distributed sizes. As a tensile stress is applied, the liquid front will pierce through pores with radius $r_{\mathrm{p}}$ if $\Delta P>2 \gamma / r_{\mathrm{p}}$, and retreats into the matrix until it meets a pore small enough to balance the pressure difference. As the stress is increased, more pores are broken through in the equilibrium configuration. Consequently, a larger area of unfilled open pores is formed in the membrane and more light is scattered at the liquid/air interface (Fig. 2a), leading to the tunable optical transparency of the composite. We demonstrate this by gradually stretching the liquid-infused membrane. Increasing the strain results in lower light transmission in visible wavelengths until the membrane finally 
becomes opaque, and the light transmission recovers when the strain is relaxed (Fig. 2b). Imaging by infrared camera, which collects the light of wavelengths $2.505 .1 \mu \mathrm{m}$, shows that the signal from a hot background $\left(50^{\circ} \mathrm{C}\right)$ is effectively scattered by the surface as it is stretched (Fig. 2c, Movie 2).

The continuous tunability of the liquid film morphology also provides a way to dynamically manipulate the mobility of both oil and water droplets on the surface. Controlling fluids, particularly those with low surface tension, has proved extremely difficult on rigid surfaces: tuning fluid mobility, which is highly sensitive to minor changes in surface geometry, notoriously requires restructuring of intricate and fragile topographical elements that are hard to change ${ }^{28,29}$. Soft surfaces show more promise, but they still fail with low-surface-tension liquids ${ }^{30}$, and, notably, droplets of any surface tension become irreversibly immobilized once they pin on and wet a solid substrate ${ }^{31}$. In our liquid-infused system, the flat, atomically smooth liquid interface in the relaxed state serves as a lubricating layer that allows drops to slide freely, ${ }^{19}$ while the undulating morphology of the stretched film reversibly halts the moving drops by pinning them on fluidic, rather than solid, textures (Fig. 3a, Supplementary Fig. S3). Figure 3b demonstrates this ability for a drop of low-surface-tension silicone oil on a tilted surface: the droplet slides on an undeformed substrate but stops and is held in place when the substrate is stretched. In the latter state, even a newly deposited oil drop is immobilized and stays where it is placed on the surface. However, as soon as the stress is released, both drops begin to slide (Movie 3). The changes in mobility correlate with reconfiguration of the film under reversible stretch, and confocal imaging further confirms that the lubricant/oil interface becomes rough when the membrane is stretched and returns to flat when the stress is relaxed (Supplementary Figs. S4-S5). Additionally, we detected no oil residue left on the membrane after the oil drop slides away upon release from pinning, consistent with the lubricating film remaining continuous rather than allowing the oil to wet and foul the underlying solid (Movie 4). This surface thus provides a simple, stimulus-responsive way to tune liquid mobility and transport in real time, not only by varying the surface wettability but by reversibly stopping and starting the motion of the droplets themselves. 
In addition to directly manipulating moving droplets, the infused surface can be designed to achieve varying stimulus-sensitivity profiles for liquids with different surface tensions. By varying the volume fraction of lubricant on a relaxed substrate, we can tune the responsiveness of the system to deformation. If the overcoating lubricant layer has a thickness $h$ that is large enough, when the substrate is deformed, the fluid will be able to fill the newly formed gaps and equalize the internal and external pressure without disrupting the flat lubricant/oil interface. For an isotropic porous matrix with a drained bulk modulus $K$ subject to a stress $\sigma$, and with the thickness of the excess lubricant layer $h$ relative to the thickness of the porous membrane $H$, the characteristic strain to just expose the porous substrate by increasing the volume of the matrix satisfies the relation $h / H \sim \sigma / 3 K$, and for stresses larger than the resulting simple threshold, liquid drops are likely to be pinned. This relationship allows the deformation sensitivity threshold to be straightforwardly tailored by varying the amount of lubricant. At the same time, for a given amount of lubricant, a lubricant/oil combination with a smaller interfacial energy allows the liquid to retreat and expose a smaller pore so that less strain will be required to disrupt the flat interface and pin droplets with lower interfacial tensions.

Indeed, Figure 3c shows the sliding angles (the tilt required for the droplet to slide) for silicone oil drops on membranes infused with different amounts of lubricant per area of membrane $\left(2.6-3.0 \mu \mathrm{L} / \mathrm{cm}^{2}\right)$ under different membrane strains $(0-10 \%)$. For a given amount of lubricant, the sliding angle increases with increasing strain, as the surface becomes rougher. For a given strain, membranes with less lubricant have higher sliding angles, consistent with less stretch leading to greater exposed roughness and pinning. To test how lubricant/oil interfacial energy influences the sensitivity to strain, we used Krytox $103\left(3.0 \mu \mathrm{L} / \mathrm{cm}^{2}\right)$ as lubricant, and compared the sliding angles as a function of strain for hydrocarbon oils of different surface energies: octane $(21.6 \mathrm{mN} / \mathrm{m})$, decane $(23.7 \mathrm{mN} / \mathrm{m})$, dodecane $(25.4 \mathrm{mN} / \mathrm{m})$ and hexadecane $(27.3 \mathrm{mN} / \mathrm{m})$. Figure $3 \mathrm{~d}$ shows that oils of lower surface tension have a sharp increase in the sliding angle when a small strain is applied. For example, a small strain change from 0 to $\sim 2 \%$ increases the sliding angle from $5^{\circ}$ to $18^{\circ}$ for a droplet of octane. The same strain change has no discernible effect on hexadecane, which 
requires $\sim 9 \%$ strain to reach a sliding angle of $\sim 18^{\circ}$. The system is thus sensitive enough to distinguish among a variety of low-surface-tension liquids

While a potentially important factor in this system is the viscosity of the infused lubricant ${ }^{32}$, we find that it does not play a dominant role in terms of the response rate to the mechanical load. This can be expected, since the flow rate of the lubricant in the porous Teflon is very fast compared to the small rate changes due to viscosity (see Supplementary Information for details, Fig. S6-S7).

Beyond planar strain, more complex and localized deformations and the associated changes in the material's properties can be induced by bending, poking, or swelling the liquid-infused elastic membrane (Fig. 4a-c, Movies 5,6). For example, sliding water drops slow down and stop when the membrane is bent, and resume moving as the membrane is released (Fig. 4d, Movies 7). Compared to stretching, bending generates a sharper response in a localized area, and thus allows the fast and efficient control of liquids, from lowsurface-energy oils to water. Figure $4 \mathrm{c}$ shows optical switching of a liquid-infused membrane attached to silicone tubing by reversible swelling/drying in chloroform. The membrane becomes opaque as the tubing expands upon swelling, and recovers after drying. The fluidic nature of the lubricant also enables the liquid-infused membrane to be used in applications such as anti-fingerprint surfaces. As shown in Figure 4e, a fingerprint left on the stretched textured surface disappears, when the strain is relaxed and the liquid flows back and fills the gaps made by the fingerprint, thus providing a dynamic stamping/screening property by allowing self-healing of the liquid layer.

Our results show that liquid-infused structured elastic solids present a conceptually different approach to creating adaptive materials that enable a single surface to reversibly span an entire range of functional properties from one extreme to another, with stimulussensitive tunability. By pairing the fluidity of an infiltrated liquid with the elastically responsive topography of a porous solid, we can bring many materials properties that are sensitive to fine features of the surface topography associated with optics, wettability, adhesivity, anti-fouling and surface transport characteristics into the realm of responsive materials. Here we used a graded mechanical stimulus to simultaneously adjust the 
surface's transparency and reversibly start and stop sliding oil droplets, but the porous solid can be designed to swell, shrink, or otherwise alter the pore sizes in response to temperature, light, magnetic or electric fields, chemical signals, pressure, or other environmental conditions. The strategy is, therefore, not only inherently multifunctional but can be generalized to a wide range of stimuli. We show that the stimulus-sensitivity level can be easily adjusted by varying the amount of fluid, and our minimal model provides a rough guide to how both the fluid and pore features can be rationally chosen to produce desired surface properties. The ability to adapt the film surface all the way from atomically flat to a range of highly tailored topographies may open opportunities for designing a new class of materials that take advantage of the diverse possibilities of both: multifunctional surfaces, such as tents that block light on a dry sunny day, but become both transparent and water-repellent on a dim rainy day; adaptive anti-ice surfaces that adjust from droplet impact resistant topographies in cold, dry weather, to a condensationpreventive smooth surface in humid conditions; fuel transport pipes or microfluidic systems that responsively control flow according to both environmental conditions and fluid identity; highly precise self-tuning optical lenses; self-cleaning textured surfaces that purge dirt buildup by a triggered shift to flat, and more.

\section{Methods}

Material fabrication. The hybrid membrane was prepared from an elastic PDMS film (0.501.5 mm thick) and the porous Teflon membrane (average pore size of $200 \mathrm{~nm}$, thickness of $\sim 45 \mu \mathrm{m}$, Sterlitech Corporation USA), by using a thin layer of PDMS oligomer as adhesive. The PDMS film was first activated by $\mathrm{O}_{2}$ plasma treatment for $10 \mathrm{~s}$. A thin layer of PDMS curing precursor (Dow Corning Sylgard 184, 10:1 base and curing agent) was then coated on the substrate, and annealed in an oven at $70^{\circ} \mathrm{C}$ for $15-20$ min to obtain a sticky oligomer. The porous Teflon membrane was placed on top with a loading pressure of $\sim 1000 \mathrm{~Pa}$. The sticky PDMS oligomer thus firmly attached the nanofiber networks to the elastic substrate. The integrated multilayer was then placed in the oven at $70^{\circ} \mathrm{C}$ for $2 \mathrm{~h}$ to ensure complete curing. To prepare the liquid-infused surface, a perfluorinated fluid 
(Dupont $^{\mathrm{TM}}$ Krytox $^{\circledR} 103$ perfluoropolyether; $\gamma=17.4 \mathrm{mN} / \mathrm{m}$ ) was added onto the asprepared membrane to form an over-coated layer. The thickness of the over-coated layer can be controlled by the fluid volume given a known surface area of the sample. The membrane stretching was carried out by a home-made uniaxial stretcher. The membrane was mounted by screwed clippers on a pair of arms of the stretcher. The evolution of the distance between the arms was measured during the experiment, which was further used to calculate the membrane strain.

Characterization. Optical transmission measurements for visible light were carried out using a fiber spectrophotometer (Ocean Optics). In-line light transmission was recorded in all measurements. Thermal imaging measurements were performed by using an IR camera (FLIR SC5000). A blackbody calibrator (OMEGA BB701) that was set at $50^{\circ} \mathrm{C}$ was used as background. The stretcher was placed between the camera and the hot background. The distance between the membrane and hot background was $4 \mathrm{~cm}$.

Oil sliding angles and contact angle hysteresis were recorded by a contact angle goniometer (KSV CAM101) at ambient conditions. The interaction between the lubricant and impinging oil was imaged using Zeiss LSM 720 laser confocal microscope and Zen software. A time-series mode was used to track the in situ evolution at the interface during stretch. A Z-stacking mode was used to capture the morphology of the lubricant/oil interface at different strains.

Simulation. The liquid pressure of a porous matrix infused with liquid under a mechanical load was calculated by using finite element simulation method. The layer of the liquidinfused matrix was modeled as a poroelastic material. Three types of mechanical loads, including tension, bending and poking, were simulated individually. The "Soil" type of solver was selected in the simulation. The instantaneous liquid pressure was calculated by applying the mechanical load in a very small time step, which is five orders of magnitude smaller than the time for diffusion to reach equilibrium. A zero flux boundary condition was also imposed on the surface of the top layer.

Further details of the methods are available in the Supplementary Information. 


\section{Acknowledgements}

The work was supported by the AFOSR MURI award FA9550-09-1-0669-DOD35CAP (optical properties) and the ONR MURI award N00014-12-1-0875. We thank T. Blough for the help in stretcher design and fabrication. We also thank M. Kolle and J. Alvarenga for the help with the optical test. We acknowledge the use of the facilities at the Harvard Center for Nanoscale Systems supported by the NSF under award ECS-0335765.

\section{Author contributions}

X.Y. and J.A. conceived the concepts of the research. J.A. supervised the research. X.Y. designed and performed the experiments. X.Y. and T.-S.W. prepared samples. Y.H. and L.M. set up models. Y.H. carried out finite element simulations. X.Y., A.G., Y.H., and J.A. wrote the manuscript. All authors contributed to revising the manuscript.

\section{Additional information}

Supplementary information is available in the online version of the paper. Reprints and permissions information is available online at www.nature.com/reprints. Correspondence and requests for materials should be addressed to J.A.

\section{Competing financial interests}

The authors declare no competing financial interests.

\section{References:}

1. Stuart, M.A.C. et al. Emerging applications of stimuli-responsive polymer materials. Nature Materials 9, 101-113 (2010).

2. Xia, F. \& Jiang, L. Bio-inspired, smart, multiscale interfacial materials. Advanced Materials 20, 2842-2858 (2008).

3. Kim, P., Zarzar, L.D., He, X.M., Grinthal, A. \& Aizenberg, J. Hydrogel-actuated integrated responsive systems (HAIRS): Moving towards adaptive materials. Current Opinion In Solid State \& Materials Science 15, 236-245 (2011).

4. Huang, Y.F. et al. Improved broadband and quasi-omnidirectional anti-reflection properties with biomimetic silicon nanostructures. Nature Nanotechnology 2, 770-774 (2007).

5. Vukusic, P. \& Sambles, J.R. Photonic structures in biology. Nature 424, 852-855 (2003). 
6. Quéré, D. Wetting and roughness. Annual Review Of Materials Research 38, 71-99 (2008).

7. Poetes, R., Holtzmann, K., Franze, K. \& Steiner, U. Metastable Underwater Superhydrophobicity. Physical Review Letters 105, 166104 (2010).

8. Arzt, E., Gorb, S. \& Spolenak, R. From micro to nano contacts in biological attachment devices. Proceedings of the National Academy of Sciences of the United States of America 100, 1060310606 (2003).

9. Qu, L.T., Dai, L.M., Stone, M., Xia, Z.H. \& Wang, Z.L. Carbon nanotube arrays with strong shear binding-on and easy normal lifting-off. Science 322, 238-242 (2008).

10. Hochbaum, A.I. \& Aizenberg, J. Bacteria Pattern Spontaneously on Periodic Nanostructure Arrays. Nano Letters 10, 3717-3721 (2010).

11. Duprat, C., Protiere, S., Beebe, A.Y. \& Stone, H.A. Wetting of flexible fibre arrays. Nature 482, 510-513 (2012).

12. Zheng, Y.M. et al. Directional water collection on wetted spider silk. Nature 463, 640-643 (2010).

13. Bettinger, C.J., Langer, R. \& Borenstein, J.T. Engineering Substrate Topography at the Micro- and Nanoscale to Control Cell Function. Angewandte Chemie-International Edition 48, 5406-5415 (2009).

14. Bocquet, L. \& Lauga, E. A smooth future? Nature Materials 10, 334-337 (2011).

15. Braun, R.J. Dynamics of the Tear Film. Annual Review of Fluid Mechanics, Vol 44 44, 267-297 (2012).

16. Thornton, D.J. \& Sheehan, J.K. From mucins to mucus: toward a more coherent understanding of this essential barrier. Proc Am Thorac Soc 1, 54-61 (2004).

17. Aizenberg, J., Tkachenko, A., Weiner, S., Addadi, L. \& Hendler, G. Calcitic microlenses as part of the photoreceptor system in brittlestars. Nature 412, 819-822 (2001).

18. Mathger, L.M., Denton, E.J., Marshall, N.J. \& Hanlon, R.T. Mechanisms and behavioural functions of structural coloration in cephalopods. Journal of the Royal Society Interface 6, S149-S163 (2009).

19. Wong, T.S. et al. Bioinspired self-repairing slippery surfaces with pressure-stable omniphobicity. Nature 477, 443-447 (2011).

20. Lafuma, A. \& Quéré, D. Slippery pre-suffused surfaces. Europhys Lett 96, 56001 (2011).

21. Kim, P. et al. Liquid-Infused Nanostructured Surfaces with Extreme Anti-Ice and Anti-Frost Performance. Acs Nano 6, 6569-6577 (2012).

22. Epstein, A.K., Wong, T.S., Belisle, R.A., Boggs, E.M. \& Aizenberg, J. Liquid-infused structured surfaces with exceptional anti-biofouling performance. Proc Natl Acad Sci U S A 109, 13182-7 (2012).

23. Quéré, D. Non-sticking drops. Reports On Progress In Physics 68, 2495-2532 (2005).

24. Seemann, R. et al. Wetting morphologies and their transitions in grooved substrates. Journal Of Physics-Condensed Matter 23, 184108 (2011).

25. Scherer, G.W. \& Smith, D.M. Cavitation during Drying of a Gel. Journal Of Non-Crystalline Solids 189, 197-211 (1995).

26. Rice, J.R. \& Cleary, M.P. Some Basic Stress Diffusion Solutions for Fluid-Saturated Elastic PorousMedia with Compressible Constituents. Reviews Of Geophysics 14, 227-241 (1976).

27. Biot, M.A. General theory of three-dimensional consolidation. Journal Of Applied Physics 12, 155-164 (1941).

28. Yao, X., Gao, J., Song, Y.L. \& Jiang, L. Superoleophobic Surfaces with Controllable Oil Adhesion and Their Application in Oil Transportation. Advanced Functional Materials 21, 4270-4276 (2011). 
29. Grigoryey, A., Tokarey, T., Kornev, K.G., Luzinov, I. \& Minko, S. Superomniphobic Magnetic Microtextures with Remote Wetting Control. Journal Of The American Chemical Society 134, 12916-12919 (2012).

30. Choi, W. et al. Fabrics with Tunable Oleophobicity. Advanced Materials 21, 2190-2195 (2009).

31. Callies, M. \& Quéré, D. On water repellency. Soft Matter 1, 55-61 (2005).

32. Smith, J.D. et al. Droplet mobility on lubricant-impregnated surfaces. Soft Matter 9, 1772-1780 (2013).

\section{Figure Legends}

Figure 1 Design of liquid-infused dynamic materials. (a) Scheme showing the central concept of the system: the topography of a liquid film on an elastic porous substrate reconfigures in response to external stimuli (for example, mechanical stretch). The porous matrix is illustrated as composed of unit pores of radius $r_{\mathrm{p}}$. Initially a thin layer of excess liquid of thickness $h$ overcoats the substrate and forms a flat interface. Under small tension, the film surface undulates. Under higher tension, the surface begins to retreat into the expanded pores. A rough surface develops with a meniscus radius $r_{\mathrm{m}}$. (b) Schematic presentation of material construction from a PDMS base layer, porous Teflon, and infused liquid (top) and the photographs of the dry and lubricated substrates (bottom). A thin layer of PDMS precursor pre-coated on the PDMS firmly adheres the base to the Teflon nanofibers with a slight penetration into the porous membrane. (c) Finite element calculation of the instantaneous pore pressure of the liquid-infused porous Teflon attached to a PDMS substrate. When a mechanical load is applied, a field of negative pore pressure is generated throughout the porous membrane. (d) Photographs demonstrating the stretch-induced surface changes resulting from liquid redistribution. Insets: Microscopic images (scale bar: $50 \mu \mathrm{m}$ ) showing the appearance of unfilled air gaps.

\section{Figure 2 Adaptive optical transmission of the liquid-infused material under mechanical}

stretch. (a) Scheme illustrating the change in optical transmission under stretch. Air pockets induced by stretching lead to increased light scattering. (b) Tunability and reversibility of in-line optical transmission measurements in the visible light range under mechanical stretch (lubricant amount per membrane area was $2.5 \mu \mathrm{L} / \mathrm{cm}^{2}$ ). The red lines indicate the stretching process and the blue lines indicate the relaxing process, respectively. (c) Images by IR camera showing the induced change in transmission in front of an unshielded hot plate (top-left) and a hot plate screened by a membrane (note the characteristic concentric rings of the plate surface and the 
unscreened regions on top and bottom of the plate). The color gradient from black to purple indicates the IR signal emission from low to high. Most of the IR signal of the hot background is scattered by the membrane upon stretching (strain $0 \%, 6.9 \%$ and $9.9 \%$ is shown).

Figure 3 Dynamic control of droplet mobility. (a) Scheme showing the control mechanism: a droplet of test liquid changes from sliding to pinning under external stimuli (for example, mechanical stretch) as the film surface reconfigures from flat to rough. (b) Demonstration of the control of oil mobility on the dynamic slippery surface under stretch. A drop of silicone oil (6 $\mu \mathrm{L}, \gamma=\sim 20.4 \mathrm{mN} / \mathrm{m}$ ) was deposited on a tilted surface, sliding down (i-iii) until a strain of $6 \%$ was applied (iv). A second oil drop deposited on the stretched surface was also pinned (v). Both drops slid down when the strain was relaxed (vi-viii). (c) Comparison of sliding angle (silicone oil) as a function of strain on the PDMS substrate with different amounts of infused lubricant. (d) Tuning the oil sliding angle as a function of strain for test droplets of different interfacial tensions. Perfluoro-lubricant (Krytox 103) with a unit amount of $3.0 \mu \mathrm{L} / \mathrm{cm}^{2}$ was used as the infused fluid.

Figure 4 Effect of different deformation mechanisms. (a) Bending and the corresponding pressure changes predicted by finite element modeling. (b) Poking and the corresponding pressure changes predicted by finite element modeling. (c) Reversible swelling and drying of an infused substrate attached to silicone tubing. (d) Comparison of water drops ( $9 \mu \mathrm{L}$, dyed with Rhodamine B) sliding on a slippery surface without (upper row) and with (lower row) bending. The membrane was tilted $\sim 60^{\circ}$. (e) Anti-fingerprint ability. The fluid flows back and fills the gaps made by the fingerprint, indicating a selfhealing mechanism. 
a

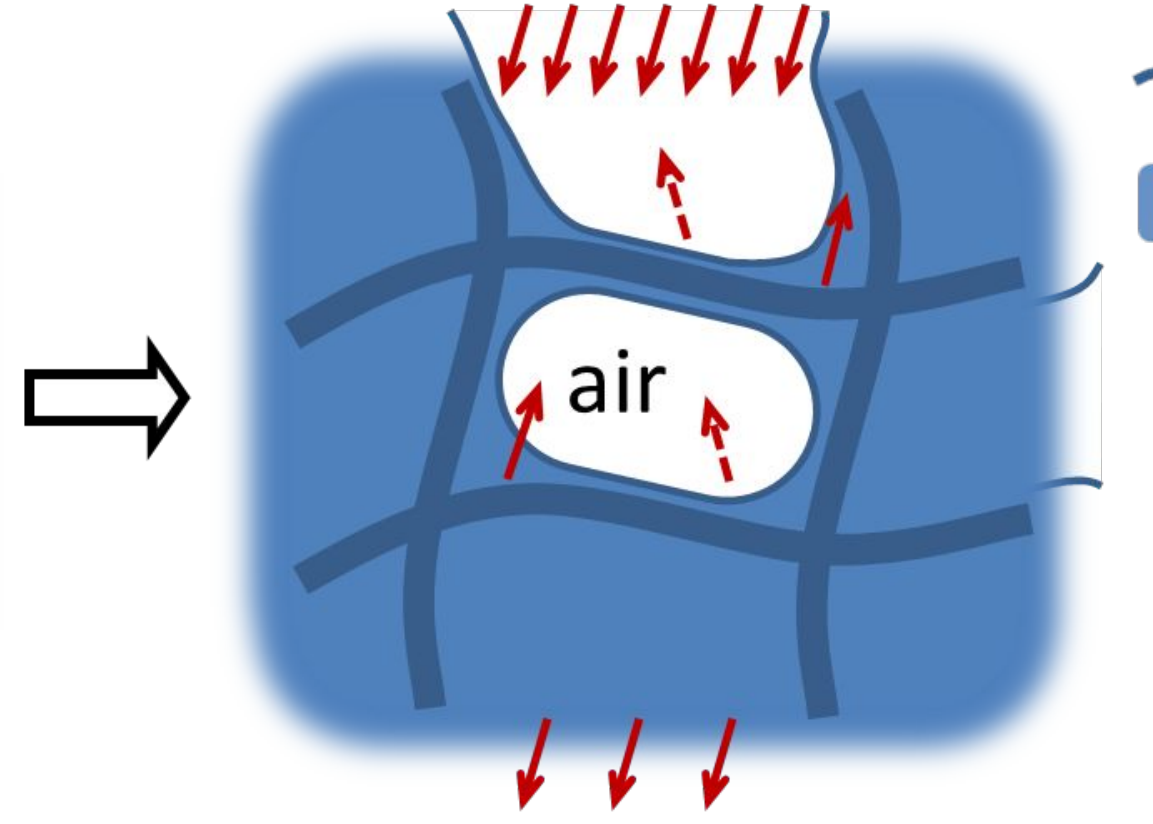

+ Porous skeleton

Infusing liquid

$\downarrow$ Incident light

$\uparrow$ Light scattering at solid/liquid interface

Light scattering at liquid/air interface

b
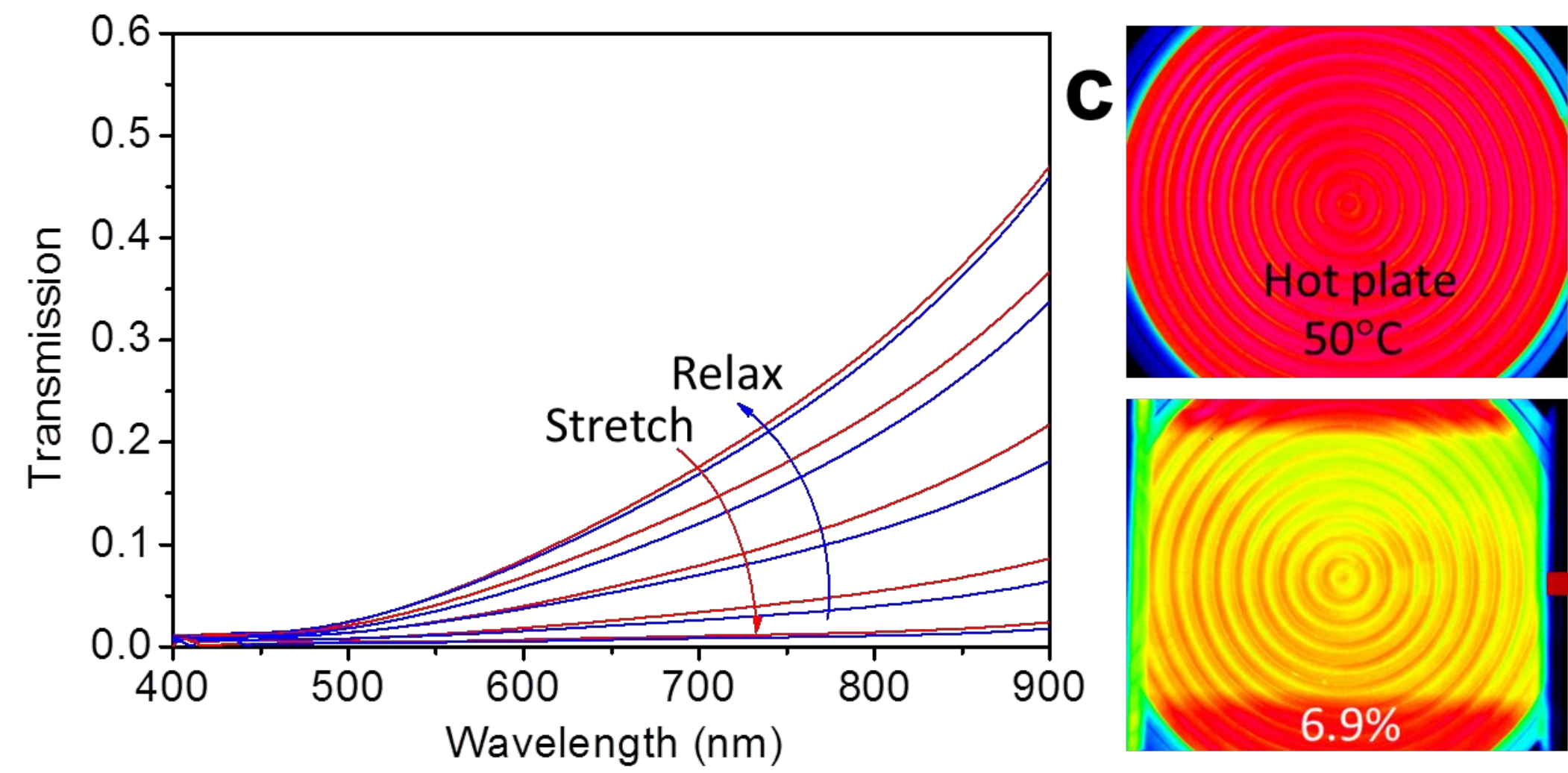

Membrane binder
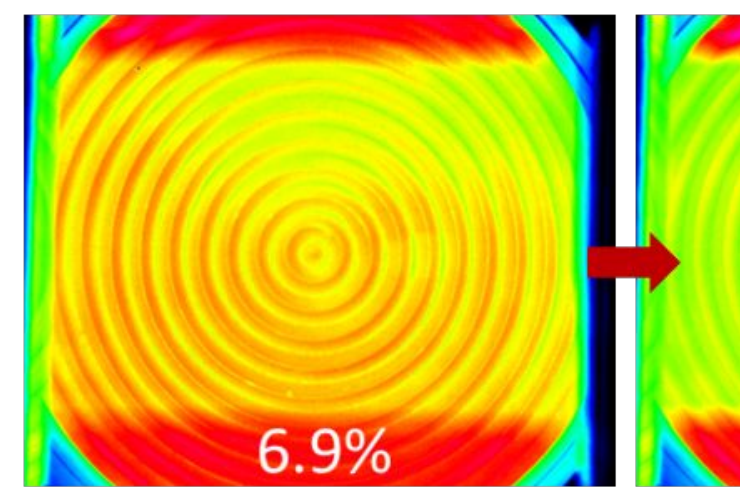

Strain 0\%

$50^{\circ} \mathrm{C}$ 

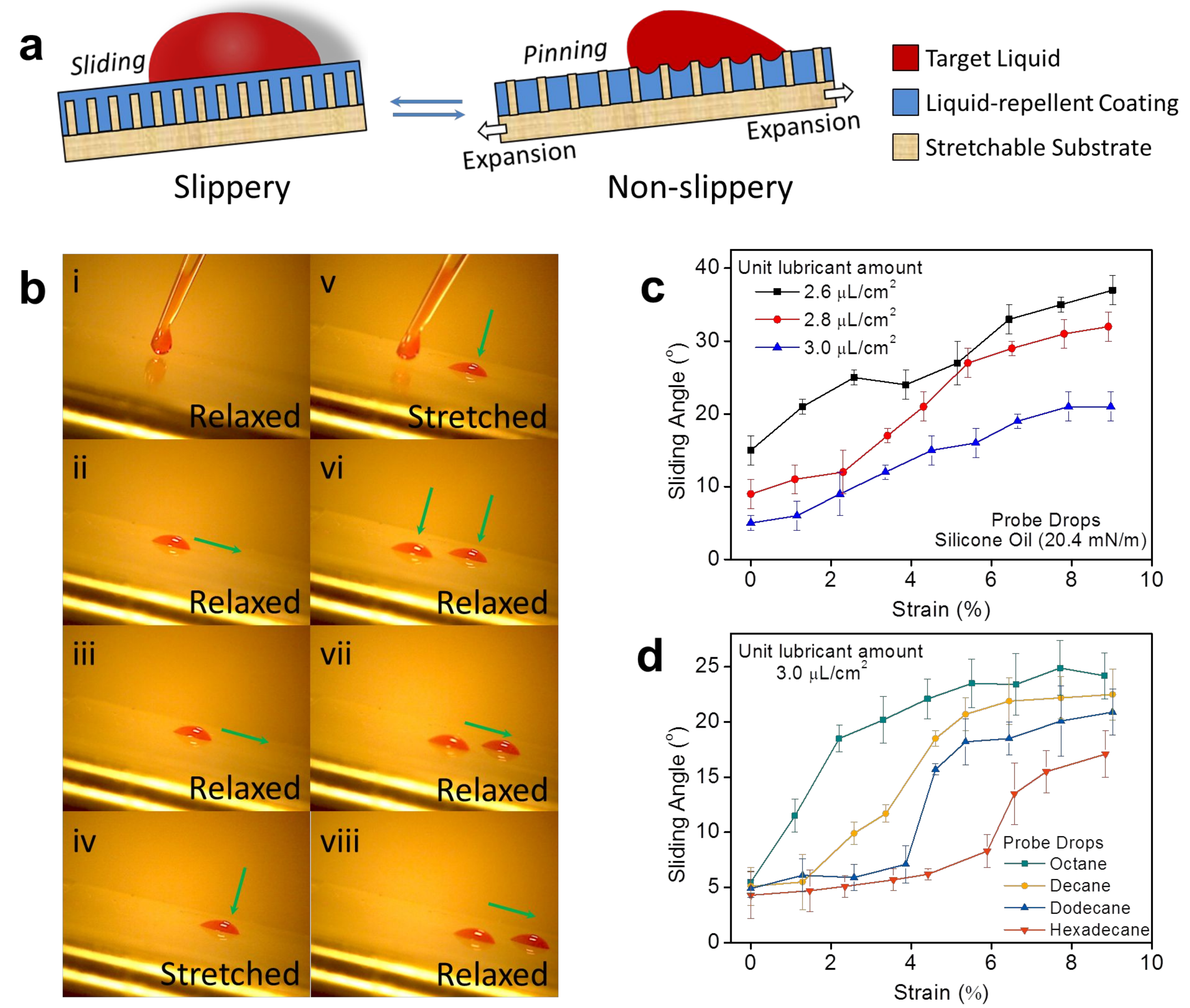
